\title{
Recherche d'anticorps dirigés contre les différents types de fibres chez le poulet
}

Chez les oiseaux domestiques, les muscles que l'on trouve dans la cuisse et les pattes sont généralement décrits comme étant de type lent oxydatif. Au contraire, les muscles pectoraux (muscles du filet) sont de typologie variable. Ils présentent une typologie myofibrillaire mixte (oxydo-glycolytique ou IIA, et glycolytique ou IIB) chez les palmipèdes et les oiseaux qui volent, et un caractère complètement glycolytique (fibres musculaires $100 \%$ IIB) chez la dinde et le poulet.

On connaît par ailleurs les liens qui peuvent exister entre la typologie des fibres musculaires et la qualité de la viande en général. Ainsi, dans les muscles présentant différents types de fibres dans des proportions variables, la présence d'une majorité de fibres de tel ou tel type a un effet sur les caractéristiques organoleptiques et technologiques de la viande. Des études récentes ont par ailleurs montré que des facteurs comme la vitesse de croissance, l'âge à l'abattage, ou la conformation des carcasses (augmentation du rendement en muscles pectoraux) pouvaient modifier la typologie des fibres dans certains muscles. D'autres facteurs génétiques, comme une plus ou moins grande susceptibilité au stress, peuvent aussi entraîner des modifications de la typologie des muscles.

Dès lors, il paraît intéressant de mieux comprendre comment les types de fibres musculaires se mettent en place chez l'embryon d'oiseau et de voir comment on pourrait maîtriser ce facteur, notamment à l'aide de la génétique. Pour cela, il semble indispensable de posséder des outils de typologie fiables et facilement utilisables en grande série. L'obtention d'anticorps spécifiquement orientés vers des types particuliers de fibres permettrait d'envisager une telle approche, notamment au travers de biopsies exploitées en immuno-histologie (simplification des techniques actuelles en coupes sériées) ou avec des méthodes ELISA.

\section{Mode opératoire}

Les muscles sélectionnés (3 au total) ont été prélevés après anesthésie et sacrifice d'un poulet standard adulte (20 semaines). Les deux muscles retenus avaient les caractéristiques suivantes (en histologie classique) :

Posterior latissimus dorsi (PLD) : fibres IIA et IIB

Pectoralis major (PM) : fibres IIB.

De plus le muscle sartorius (SART, fibres I, IIA et IIB) a été conservé pour l'analyse histologique.
Nous avons testé 70 anticorps issus des séries S4 (poisson + dinde), S5 (poulet + porc) et S2 (bovin + cheval).

L'ensemble des observations a été fait sur des coupes congelées ( $7 \mu \mathrm{m}$ d'épaisseur). Après un rinçage rapide dans du PBS, la coupe est mise à incuber pendant 10 minutes à température ambiante en présence de sérum normal (4\% dans du PBS) afin de bloquer les sites non spécifiques. Les coupes sont ensuite mises à incuber (chambre humide à + $4{ }^{\circ} \mathrm{C}$ ) pendant une nuit en présence du surnageant à tester. Après deux rinçages dans du PBS, l'anticorps secondaire (FITC) est appliqué pendant une heure à température ambiante, puis les coupes sont rincées abondamment au PBS et montées en milieu aqueux. L'observation au microscope est réalisée immédiatement afin de réduire d'éventuels problèmes issus de l'extinction naturelle de la fluorescence.

\section{Résultats de la première production d'anticorps}

Dans un premier temps 5 groupes ont été constitués selon l'efficacité de marquage des différents anticorps sur les muscles PLD, PM et SART des poulets adultes (tableau 1).

Nous avons également testé, dans les mêmes conditions, des anticorps produits par l'équipe de Schiaffino, ce testage s'est avéré de peu d'intérêt chez l'espèce poulet.

Parmi tous les anticorps, 7 ont été retenus (S5 10H5, S5 12D1, S5 16D12, S5 8F3, S4 14B12, S4 9H5 ET S4 17F7) qui paraissaient avoir un intérêt plus particulier. Ils ont donc été testés une nouvelle fois à des dilutions plus importantes $(1 / 10,1 / 100$ et $1 / 1000)$ afin de s'assurer de leur spécificité. Ces résultats obtenus en histologie ont été confrontés à ceux obtenus par la technique du Westernblot par la société Biocytex. Les résultats sont présentés dans le tableau 2.

Pour trois anticorps, il y a une différence nette entre les résultats des deux techniques. En effet, l'anticorps S4 9H5, par exemple, marque les fibres de type IIB en immunohis-

Tableau 1. Résultats du testage des anticorps sur les muscles de poulets.

\begin{tabular}{|c|l|c|}
\hline Groupe & Marquage & Proportion d'anticorps \\
\hline G1 & Pas de marquage & $19 / 70$ soit $27 \%$ \\
G2 & Marquage de toutes les fibres rapides & $36 / 70$ soit $51 \%$ \\
G3 & Marquage de toutes les fibres & $9 / 70$ soit $13 \%$ \\
G4 & Marquage non homogène (mosaïque) & $5 / 70$ soit $7 \%$ \\
G5 & Marquage du tissu conjonctif & $1 / 70$ soit $1 \%$ \\
\hline
\end{tabular}

H. RÉMIGNON*, V. DESROSIERS

INRA Station de Recherches Avicoles 37380 Nouzilly.

* Adresse actuelle $\square$. Ecole Nationale Supérieure Agronomique de Toulouse, BP 107, 31326 CastanetTolosan Cedex 
Tableau 2. Confrontation des résultats de spécificité de certains anticorps en fonction de la technique d'identification des types de fibres utilisée.

\begin{tabular}{|l|c|c|l|}
\hline Anticorps & Histologie $^{(1)}$ & Western-blot & \multicolumn{1}{|c|}{ Observations } \\
\hline S4 9H5 & IIB & - & Non concordance Western - histologie \\
S4 14B12 & Anti-rapide & + & Anticorps anti-fibre rapide \\
S4 17F7 & Anti-lent & - & Anticorps anti-fibre lente \\
S5 8F3 & IIA et quelques IIB & - & Non concordance Western - histologie \\
S5 10H5 & Anti-rapide & - & Non concordance Western - histologie \\
S5 12D1 & Anti-rapide & + & Anticorps anti-fibre rapide \\
S5 16D12 & Anti-rapide & + & Anticorps anti-fibre rapide \\
\hline
\end{tabular}

(1) - = Négatif : absence de marquage ; + = Positif : présence de marquage.

(2) Test effectué sur le muscle posterior latissimus dorsi (PLD).

tologie mais donne un résultat négatif lors du testage en Western-blot. Or le muscle PLD, qui a été utilisé en Western-blot, est toujours décrit comme contenant des fibres de type IIA et IIB chez le poulet. Le même type de remarque peut être fait pour les anticorps S5 8F3 et S5 10H5. Ces écarts peuvent être expliqués par une éventuelle modification structurelle de la myosine au cours de son extraction chimique ou de l'électrophorèse séparative.

Par la suite, seuls les anticorps suivants ont été retenus car ils présentaient un réel intérêt en immunohistologie et en Western-blot:

S4 17F7 anti-fibre I

S5 16D12 anti-fibre rapide

S5 8F3 anti-fibre IIA (et certaines IIB)

S4 9H5 anti-fibre IIB.

\section{Résultats de la deuxième production d'anticorps}

Ces 4 anticorps ont fait l'objet d'une nouvelle production que nous avons testée dans des conditions identiques à celles présentées précédemment. Les résultats ont été quelque peu modifiés par rapport aux précédents car le marquage des fibres I (S4 17F7) a beaucoup faibli en intensité et ceux des anticorps S5 16D12 et S5 8F3 ont disparu. Par contre le marquage anti-IIB du S4 9H5 a persisté correctement. Pour essayer de corriger ces écarts de révélation, les nouvelles productions d'anticorps ont été utilisées à des dilutions plus faibles $(1 / 10$ et $1 / 100)$ et avec des systèmes de révélation différents (Kit LSAB de Dako, Kit Vectastain de Vector Laboratories). Mais les résultats n'ont pas été améliorés et seuls les anticorps S4 17F7 (anti-I) et S4 9H5 (antiIIB) ont été retenus pour de nouveaux tests. Ces tests supplémentaires ont été effectués sur des individus et des muscles différents de ceux utilisés précédemment, mais dans les mêmes conditions que lors du criblage primaire (utilisation de l'anticorps dilué au 1/1000 et révélation par le FITC (Sigma) au 1/500). Les muscles utilisés chez les différents animaux étaient le posterior latissimus dorsi (PLD, fibres IIA et IIB), le pectoralis major (PM, fibres IIB), le sartorius (SART) et l'adducteur profond (AP) qui contiennent tous les deux des fibres I, IIA et IIB. Les animaux utilisés étaient tous issus de la même souche et ont été testés aux âges de $1(\mathrm{n}=4), 3(\mathrm{n}=4), 6$ $(n=4) 12(n=4)$ et $20(n=3)$ semaines.

Chez l'ensemble de ces individus, l'anticorps S4 17F7 (anti-I) fonctionne correctement dans l'ensemble des muscles où des fibres lentes sont présentes. On note cependant une intensité de marquage plus ou moins forte suivant les âges, le marquage paraissant plus intense à partir de 3 semaines.

Pour l'anticorps S4 9 H5 (anti-IIB), les résultats sont beaucoup moins facilement interprétables. Ainsi, on obtient $100 \%$ de marquage correct chez 2 individus sur 3 à l'âge de 20 semaines et simplement $83 \%$ de marquage correct chez le troisième. Dans ce dernier cas, $17 \%$ des fibres identifiées comme étant IIB en histologie classique ne sont pas reconnues par l'anticorps. La proportion de fibres correctement marquées varie ensuite beaucoup selon l'âge, mais se situe toujours autour de 60 à $70 \%$. Ces chiffres sont en fait le résultat des moyennes calculées sur tous les individus d'un même âge pour les muscles PLD, SART et AP. De plus, chez certains individus, l'efficacité du marquage pour cet anticorps varie en fait de $75 \%$ à $100 \%$ (pour des champs différents pris dans le même muscle) dans le muscle SART à 3 semaines et dans le PLD à 6 semaines.

Dans le muscle PM, au contraire, le marquage des fibres est totalement efficace, sauf à l'âge de 1 semaine où l'efficacité est voisine de $85 \%$. Cette hétérogénéité est aussi constatée dans les autres muscles ; elle est probablement due à un typage non homogène des fibres IIB à cet âge.

\section{Conclusion}

De façon générale, on ne peut pas retenir une complète spécificité de marquage de l'anticorps S4 9H5 et ce d'un âge ou d'un muscle à l'autre.

Il faut en conclure que :

- soit l'anticorps reconnaît un épitope qui n'est pas représenté à $100 \%$ dans toutes les fibres IIB de tous les poulets testés, c'est-àdire que l'anticorps reconnaît une sous-classe 
de fibres particulières que l'on ne distingue pas des IIB en histologie classique ;

- soit la technique d'identification des fibres IIB (ATPase acide classique) est imparfaite et ne permet pas de systématiquement bien typer les fibres musculaires.

Il est bien difficile de choisir entre ces deux hypothèses car on sait que certaines fibres existent mais ne sont pas détectables en histologie classique (cas des fibres IIx chez le porc). On sait aussi que la typologie myofibrillaire est dans un état dynamique (avec des possibili- tés de passage d'un type à l'autre) et qu'il peut donc aussi y avoir des fibres dans un état intermédiaire de façon transitoire et normale.

Quoiqu'il en soit, il paraît impossible d'essayer d'utiliser ces anticorps aujourd'hui en substitution des techniques ATPasiques classiques. De plus la perte totale de réactivité des anticorps S5 16D12, S5 8F3 et la diminution apparente de l'affinité du S4 17F7 sont encore un point d'interrogation supplémentaire sur leur spécificité réelle pour les types de fibres initialement identifiées.

\section{Anticorps anti-chaînes lourdes de myosine : outils d'étude de la régénération musculaire dans l'espèce dinde}

\section{Y. CHEREL, L. GUIGAND, M. WYERS}

INRA URA 703

Développement et Pathologie du tissu musculaire, École Nationale Vétérinaire, BP 40706, 44307 Nantes Cedex 03

\begin{abstract}
La production de dindes de souche médiumalourdie (mâles de 16 semaines abattus à $12 \mathrm{~kg}$ environ et femelles de 12 semaines abattues à $7 \mathrm{~kg}$ ) fournit des carcasses utilisées en découpe. Les produits issus de cette découpe ont des valeurs commerciales différentes : les pectoraux procurent une viande blanche (correspondant à des muscles rapides) transformée en escalopes ou rôtis, c'est la partie noble ; les cuisses et, dans une moindre mesure, les ailes sont composées de viande rouge, correspondant à des muscles mixtes rapides et lents. Ils sont à l'origine de steak, osso bucco et blanquette. Actuellement, la transformation de la viande pour fournir des produits de charcuterie est encore peu développée, même si cette transformation semble représenter un débouché d'avenir.
\end{abstract}

Les notions de qualité de la viande de dinde se résument aujourd'hui à deux problématiques différentes : la première concerne la couleur des muscles blancs (hétérogénéité de couleur ainsi que grisaillement) c'est-à-dire quasi-exclusivement les muscles pectoraux ; la seconde a trait à l'exsudation des viandes après transformation. Or, les muscles pectoraux sont des muscles homogènes rapides, formés presque totalement de fibres de type IIB. La production d'anticorps permettant de discriminer les chaînes lourdes de myosine (MHC) des types IIa et IIb dans le but de typer les fibres musculaires n'a donc pas, dans l'espèce dinde, l'intérêt qu'elle peut avoir en termes de qualité des viandes et de sélection dans d'autres espèces.

En revanche, cette production d'anticorps présente un intérêt en tant qu'outil d'étude de la biologie du tissu musculaire. En effet, pour l'étude de la croissance du tissu musculaire aussi bien que pour l'étude de sa régénération après lésion, l'expression des isoformes de myosine est un excellent marqueur de la différenciation cellulaire.

$\mathrm{Au}$ cours de la croissance, les fibres musculaires multinucléées fusionnent avec des myoblastes issus de la prolifération de cellules satellites. Le nombre de noyaux des fibres croît, ce qui permet, par l'intermédiaire des synthèses de myofilaments, d'augmenter la taille de la cellule, car le rapport nucléo-cytoplasmique est constant dans un type de fibre donné.

Après lésion de la fibre musculaire, les zones nécrosées sont éliminées par phagocytose macrophagique, puis les cellules satellites fournissent les myoblastes qui fusionnent avec la fibre blessée ou fusionnent entre eux pour fournir des néo-myotubes.

Après la fusion, ces néo-myotubes ou certaines portions de fibres matures synthétisent les protéines contractiles avec une succession d'isoformes qui reproduit le décours temporel observé pendant le développement embryonnaire. La mise en évidence de ces isoformes est un moyen d'étude de la différenciation cellulaire et de l'action de facteurs modulant le développement du tissu.

Nous utilisons au laboratoire, en immunofluorescence ou en immunohistoenzymologie, plusieurs anticorps anti-isomyosines développementales dans le cadre de travaux sur la régénération musculaire, et en immunocytochimie sur culture de cellules satellites pour caractériser les populations de cellules. La possession d'anticorps anti-MHC IIa et IIb compléterait de façon très intéressante la gamme existante. 